Classification
Physics Abstracts
$5.620-7.480-9.150$

\title{
COLLAPSE OF A POLYMER CHAIN IN POOR SOLVENTS
}

\author{
P. G. DE GENNES
}

Collège de France, 75231 Paris Cedex 05, France

(Reçu le 29 novembre 1974, accepté le 10 janvier 1975)

\begin{abstract}
Résumé. - Le comportement d'une chaîne flexible unique, près de la température $\Theta$ de Flory, est analysé à partir d'une analogie avec un problème magnétique. La transition vers un état globulaire compact est relié aux propriétés d'un point tricritique dans le problème magnétique.
\end{abstract}

\begin{abstract}
The behavior of a single, flexible polymer chain near the Flory temperature $\Theta$ is analysed in analogy with a magnetic phase transition. The transition to a collapsed state is related to a tricritical point for the magnetic problem.
\end{abstract}

A solute polymer chain expands in a good solvent, and collapses in a poor solvent [1]. By suitable changes in temperature, or in solvent composition, it is possible to cross over from one behavior to the other. In experiments on solutions, a demixing process replaces the collapse [1]. However, the transition from an extended shape to a globular form, for a single coil, may be of some interest for studies on protein folding [2] ; it is also of theoretical interest as a first step towards the understanding of micellar structures.

The collapse has been studied by numerical methods on lattice models, where the polymer chain is described as a self avoiding walk, and attractive interactions between neighboring monomers are added : the results have been reinterpreted recently by Domb [3]. In the present note, we present a slightly different approach, based on an analogy with the phase transitions of a magnetic system, which is already known to be useful for the simple excluded volume problem [4].

1. Self consistent field approximation. - As usual, it is helpful to start by a simple calculation of the Flory type [1] suitably extended to cover collapsed situations. This is based on the following free energy

$$
\begin{aligned}
\frac{F}{k_{\mathrm{B}} T}=3\left[\alpha^{2} / 2\right. & -\ln \alpha]+ \\
& +\frac{N}{2}\left[\rho W_{1}(T)+\rho^{2} W_{2}(T)+\cdots\right] .
\end{aligned}
$$

Here $\alpha=R / R_{0}$ is the expansion factor, and

$$
R_{0}=N^{1 / 2} a
$$

is the r.m.s. radius of an ideal coil. $N$ is the number of monomers along the chain. The average monomer concentration in the coil is

$$
\rho=k N / R^{d}=k a^{-d} N^{1-d / 2}
$$

where $d$ is the dimensionality and $k$ a numerical constant. The parameters $W_{1}, W_{2}, \ldots$ are essentially the successive virial coefficients of the monomer interaction. $W_{1}(T)$ is the excluded volume coefficient and has the form

$$
W_{1}(T)=v \frac{T-\Theta}{\Theta}\left(\left|\frac{T-\Theta}{\Theta}\right| \ll 1\right) .
$$

Here $\Theta$ is the Flory compensation temperature. (To be specific we assume $v>0$.) The second coefficient $W_{2}$ may be taken as positive and temperature independent in the range of interest. It is important to realise that both $W_{1}$ and $W_{2}$ are relevant for a discussion of the region $T=\Theta$ in three space dimensions. For $T \sim \Theta, \alpha \sim 1$ and the $W_{2}$ term is of order $N^{3-d}$ : this is to be compared with the extensional term $\frac{1}{2} \alpha^{2}-\ln \alpha$ which is of order unity. Thus $W_{2}$ is relevant for $d \leqslant 3$. The higher terms $\left(W_{3}, W_{4}, \ldots\right)$ are irrelevant for $d=3$.

Specializing now to $d=3$, retaining only $W_{1}$ and $W_{2}$ in eq. (1) inserting the form (2) for $\rho$, and minimizing the free energy, one arrives at the following amended form of the Flory equation [1] :

$$
\alpha^{5}-\alpha^{3}-\frac{y}{\alpha^{3}}=k N^{1 / 2} \frac{W_{1}}{a^{3}}=x
$$

where $y=k^{2} W_{2} a^{-6}$ is a dimensionless parameter. [Physically, $y$ can be increased by adding side groups to the chain; it can be decreased by choosing chains which are somewhat rigid.] A plot of $\alpha(x)$ for various 
values of $y$ is shown on figure 1 . For good solvents $(x \gg 1)$ the Flory curve is maintained and $R \sim N^{3 / 5}$. For poor solvents $(x \ll 0)$ we have a strong collapse and

$$
R \sim\left[N \frac{\Theta}{-T+\Theta}\right]^{1 / 3}
$$

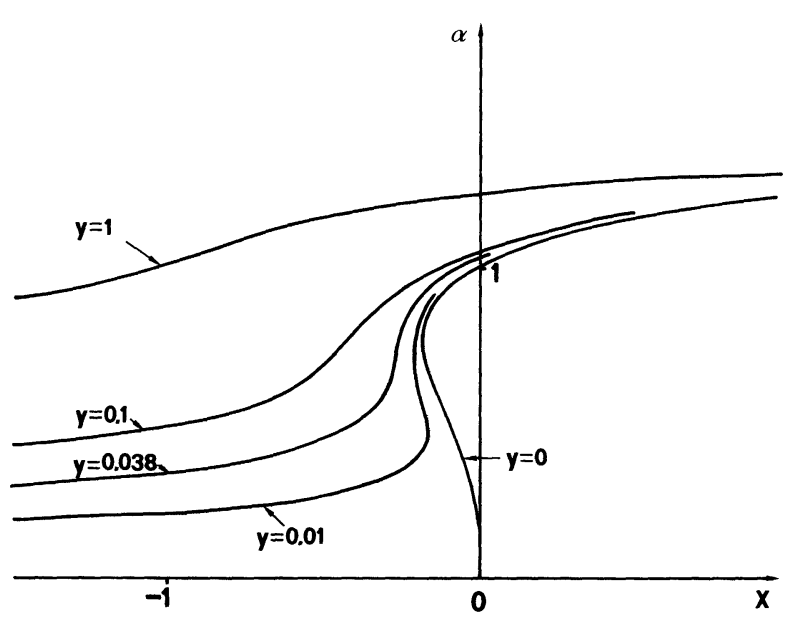

Fig. 1. - Size of a polymer chain as a function of reduced temperature in an extended Flory approximation (eq. (4)). The curves are shown for various values of a parameter $y$, which is large if the chain has some side groups, and small if the chain is slightly stiff.

For $|x| \sim 1$ (crossover region) we find two regimes depending on the value of the constant $y$. If $y>0.038$ the coil contracts smoothly upon cooling. But if $y<0.038$ eq. (4) predicts an unstable branch; if this were taken literally, it would imply an abrupt transition to the collapsed state, at a certain critical value of $x$ for which the free energies on the two (locally) stable branches become equal. In fact, since we are dealing with a finite system, the sharp transition may be an artefact of the self consistent field method : but it is probably indicative of a very steep slope, as is indeed confirmed by numerical experiments [3]. The main weakness of the latter is that they are usually performed at one single value of $y$, depending on the particular model chosen.

A more elaborate (local) version of this self consistent field method has been considered by Edwards [5] (without the $W_{2}$ term) and by Lifschitz [6] (with a saturation term); the Lifschitz results are very similar to those deduced from eq. (4).

\section{Relation between collapse and a tricritical point. -} The interaction terms in eq. (1) can be used as a starting point for a rigorous theory of chain conformations, just as the Landau free energy $F_{\mathrm{L}}[7]$ can be used as a weight function for calculations on critical points [8]. Let us consider in fact a magnetic system with magnetisation $M$, temperature $\tau$ and Landau free energy :

$F_{\mathrm{L}}=\frac{1}{2} r_{0} M^{2}+U_{1} M^{4}+U_{2} M^{6}+\cdots+\frac{1}{2}(\nabla M)^{2}$.

[Where $r_{0} \sim\left(\tau-\tau_{0}\right), \tau_{0}$ being the mean field transition point.]

There is a correspondence between the diagrams for the correlation function $\langle M(0) M(r)\rangle$ of the magnetic problem (in zero field, and above the transition point, $\tau>\tau_{\mathrm{c}}$ ) and the statistical weight for a chain extending from 0 to $r$ [4]. The bare vertex coefficients $U_{1}, U_{2}, \ldots$ are essentially identical (apart from normalization coefficients) to the virial coefficients $W_{1}, W_{2} \ldots$ The polymer length $N$ is related to $\tau$ by a Laplace transform, but from dimensional considerations on a single chain it suffices to say that $N \sim\left(\tau-\tau_{\mathrm{c}}\right)^{-1}$. We are interested here in the limit $N \rightarrow \infty\left(\tau \rightarrow \tau_{\mathrm{c}}\right)$.

The diagrams for the two problems coincide provided that certain closed loops, present in the magnetic case but absent for the single chain, are eliminated : this is achieved formally by setting the number $n$ of components of the vector $M$ equal to zero [4].

The standard excluded volume problem corresponds to a strongly positive $W_{1}$ (or $U_{1}$ ) : in this regime $U_{2}, U_{3}, \ldots$ are irrelevant. The problem discussed here corresponds to $W_{1}$ small : i.e. to a tricritical point [8]. An assumption of generalised scaling then leads to a coil radius (or correlation length) of the form

$$
\begin{aligned}
& R=a N^{v_{\mathrm{t}}} f(\tilde{x}, y) \\
& \tilde{x}=\frac{\tilde{W}_{1}}{a^{3}} N^{\Phi_{\mathrm{t}}} .
\end{aligned}
$$

Here $\tilde{W}_{1}$ is defined as in eq. (3), but with a shifted $\Theta$ point : $\widetilde{\Theta}=\Theta\left[1-g(y) N^{-\Phi_{t}}\right]$.

$v_{t}$ is a tricritical exponent defined as in reference [8]. The constant $\Phi_{t}$ is a cross-over exponent. For

$$
|\tilde{x}| \ll 1
$$

the function $f$ remains finite and $R \sim N^{v_{t}}$. For $\tilde{x}>1, f$ becomes independent of $y$ and proportional to a power of $x$; then $R \sim N^{v}$ where $v$ is the excluded volume exponent. For $\tilde{x}<-1, f$ is strongly dependent on $y$ and again behaves like a power of $|\tilde{x}|$ :

$$
\left.\begin{array}{rl}
f(\tilde{x}, y) & =|\tilde{x}|^{-\mu} b(y) \\
R & \cong N^{v_{t}-\Phi_{\mathrm{t}} \mu}\left|W_{1}\right|^{-\mu} \cong N^{v_{\mathrm{c}}}\left|W_{1}\right|^{-\mu} \\
\mu & =\left(v_{\mathrm{t}}-v_{\mathrm{c}}\right) / \Phi_{\mathrm{t}}
\end{array}\right\} .
$$

Eq. (1) suggests that the exponent $v_{\mathrm{c}}$ in the collapsed state is equal to $1 / d$ : for fixed (negative) $W_{1}$ the density $\rho$ is finite and the interaction terms $($ of order $N$ ) 
dominate the free energy : the extensional term (of order 1) can be omitted. This conclusion for $v_{\mathrm{c}}$ is probably valid even beyond the self consistent field approximation.

For $d=3$ the tricritical exponents have the mean field values $v_{t}=\Phi_{t}=\frac{1}{2}$. However, this is a marginal case (confluence of two fixed points in the renormalisation group approach) and corrections involving $(\ln N)$ are expected [9]. For $d=2$ an approximate value of $v_{t}$ may be extracted from the results of an expansion in powers of $\varepsilon=3-d$, carried to order $\varepsilon^{2}[10]:$

$$
\left.\begin{array}{rl}
v_{\mathrm{t}} & \left.=\frac{1}{2}+\left.\frac{1}{3} \frac{(n+2)(n+4)}{(3 n+22)^{2}} \varepsilon^{2}\right|_{n=0, \varepsilon=1}\right\} \\
& \cong 0.5055
\end{array}\right\}
$$

The exponent $\Phi_{\mathrm{t}}$ has not been calculated for $n=0$. But the trend is clear : even for two dimensions, the tricritical exponents are very close to the mean field values.

\section{References}

[1] See for instance Flory, P. J., Principles of polymer chemistry, (Cornell University Press, NY), 5th ed. 1966.

StOCKMAYeR, W. A., Makromol. Chemie 35 (1960) 54.

[2] See for instance LiQuori, A. M., Quartely Reviews of Biophys. 2 (1969) 1.

[3] Dомв, C., Polymer 15 (1974) 259.

See also Rapaport, D. C., Phys. Lett. 48A (1974) 339.

[4] De Gennes, P. G., Phys. Lett. 38A (1972) 339.

See also des Cloizeaux, J., J. Physique 36 (1975) no 4 (to be published).

[5] EDWARDS, S. F., in Critical phenomena eds. M. S. Green and
J. V. Sengers (Nat. Bur. Stand. Miscell. Pub.) 273 (1966) p. 225.

[6] LifSHITZ, I. M., Sov. Phys. JETP 28 (1969) 1280. See also EIZNER, Polymer Science USSR 11 (1969) 409; 14 (1972) 1965

[7] Landau, L., Lifshitz, I. M., Statistical Physics, Chap. 14 (Pergamon Press London) 1958.

[8] Griffiths, R. B., Phys. Rev. B 7 (1973) 545.

[9] Riedel, E., Wegner, F., Phys. Rev. Lett. 29 (1972) 349.

[10] Stephen, M., McCauley, J., Phys. Lett. 44A (1973) 89. 